PROCEEDINGS OF THE

AMERICAN MATHEMATICAL SOCIETY

Volume 136, Number 12, December 2008, Pages 4151-4156

S 0002-9939(08)09461-6

Article electronically published on July 23, 2008

\title{
A NOTE ON FINITE ABELIAN GERBES OVER TORIC DELIGNE-MUMFORD STACKS
}

\author{
YUNFENG JIANG \\ (Communicated by Ted Chinburg)
}

\begin{abstract}
Any toric Deligne-Mumford stack is a $\mu$-gerbe over the underlying toric orbifold for a finite abelian group $\mu$. In this paper we give a sufficient condition so that certain kinds of gerbes over a toric Deligne-Mumford stack are again toric Deligne-Mumford stacks.
\end{abstract}

\section{INTRODUCTION}

Let $\boldsymbol{\Sigma}:=(N, \Sigma, \beta)$ be a stacky fan of $\operatorname{rank}(N)=d$ as defined in [4]. If there are $n$ one-dimensional cones in the fan $\Sigma$, then modelling the construction of toric varieties [5], [6], the toric Deligne-Mumford stack $\mathcal{X}(\boldsymbol{\Sigma})=[Z / G]$ is a quotient stack, where $Z=\mathbb{C}^{n}-V$, the close subvariety $V \subset \mathbb{C}^{n}$ is determined by the ideal $J_{\Sigma}$ generated by $\left\{\prod_{\rho_{i} \nsubseteq \sigma} z_{i}: \sigma \in \Sigma\right\}$ and $G$ acts on $Z$ through the map $\alpha: G \longrightarrow\left(\mathbb{C}^{\times}\right)^{n}$ in the following exact sequence determined by the stacky fan (see [4]):

$$
1 \longrightarrow \mu \longrightarrow G \stackrel{\alpha}{\longrightarrow}\left(\mathbb{C}^{\times}\right)^{n} \longrightarrow T \longrightarrow 1 \text {. }
$$

Let $\bar{G}=\operatorname{Im}(\alpha)$. Then $[Z / \bar{G}]$ is the underlying toric orbifold $\mathcal{X}\left(\boldsymbol{\Sigma}_{\text {red }}\right)$. The toric Deligne-Mumford stack $\mathcal{X}(\boldsymbol{\Sigma})$ is a $\mu$-gerbe over $\mathcal{X}\left(\boldsymbol{\Sigma}_{\text {red }}\right)$.

Let $\mathcal{X}(\boldsymbol{\Sigma})$ be a toric Deligne-Mumford stack associated with the stacky fan $\boldsymbol{\Sigma}$. Let $\nu$ be a finite abelian group, and let $\mathcal{G}$ be a $\nu$-gerbe over $\mathcal{X}(\boldsymbol{\Sigma})$. We give a sufficient condition so that $\mathcal{G}$ is also a toric Deligne-Mumford stack. We have the following theorem:

Theorem 1.1. Let $\mathcal{X}(\boldsymbol{\Sigma})$ be a toric Deligne-Mumford stack with stacky fan $\boldsymbol{\Sigma}$. Then every $\nu$-gerbe $\mathcal{G}$ over $\mathcal{X}(\boldsymbol{\Sigma})$ is induced by a central extension

$$
1 \longrightarrow \nu \longrightarrow \widetilde{G} \longrightarrow G \longrightarrow 1
$$

i.e., we have a Cartesian diagram:

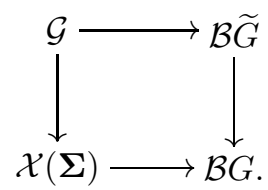

Received by the editors September 11, 2006, and, in revised form, May 8, 2007, June 10, 2007, October 11, 2007, and November 6, 2007.

2000 Mathematics Subject Classification. Primary 14A20.

Key words and phrases. Gerbes, toric Deligne-Mumford stacks.

(C)2008 American Mathematical Society Reverts to public domain 28 years from publication 
In general, the $\nu$-gerbe $\mathcal{G}$ is not a toric Deligne-Mumford stack. But if the central extension is abelian, then we have:

Corollary 1.2. If the $\nu$-gerbe $\mathcal{G}$ is induced from an abelian central extension, then it is a toric Deligne-Mumford stack.

This small note is organized as follows. In Section 2 we construct the new toric Deligne-Mumford stack from an abelian central extension and prove the main results. In Section 3 we give an example of a $\nu$-gerbe over a toric Deligne-Mumford stack.

In this paper, by an orbifold we mean a smooth Deligne-Mumford stack with trivial stabilizers at the generic points.

\section{THE PROOF OF THE MAIN RESULTS}

We refer the reader to 4 for the construction and notation of toric DeligneMumford stacks. For the general theory of stacks, see [2].

Let $\boldsymbol{\Sigma}:=(N, \Sigma, \beta)$ be a stacky fan. From Proposition 2.2 in [4, we have the following exact sequences:

$$
\begin{gathered}
0 \longrightarrow D G(\beta)^{*} \longrightarrow \mathbb{Z}^{n} \stackrel{\beta}{\longrightarrow} N \longrightarrow \operatorname{Coker}(\beta) \longrightarrow 0, \\
0 \longrightarrow N^{*} \longrightarrow \mathbb{Z}^{n} \stackrel{\beta^{\vee}}{\longrightarrow} D G(\beta) \longrightarrow \operatorname{Coker}\left(\beta^{\vee}\right) \longrightarrow 0,
\end{gathered}
$$

where $\beta^{\vee}$ is the Gale dual of $\beta$. As a $\mathbb{Z}$-module, $\mathbb{C}^{\times}$is divisible, so it is an injective $\mathbb{Z}$-module and hence the functor $\operatorname{Hom}_{\mathbb{Z}}\left(-, \mathbb{C}^{\times}\right)$is exact. We get the exact sequence:

$$
\begin{gathered}
1 \longrightarrow \operatorname{Hom}_{\mathbb{Z}}\left(\operatorname{Coker}\left(\beta^{\vee}\right), \mathbb{C}^{\times}\right) \longrightarrow \operatorname{Hom}_{\mathbb{Z}}\left(D G(\beta), \mathbb{C}^{\times}\right) \longrightarrow \operatorname{Hom}_{\mathbb{Z}}\left(\mathbb{Z}^{n}, \mathbb{C}^{\times}\right) \\
\longrightarrow \operatorname{Hom}_{\mathbb{Z}}\left(N^{*}, \mathbb{C}^{\times}\right) \longrightarrow 1 .
\end{gathered}
$$

Letting $\mu:=\operatorname{Hom}_{\mathbb{Z}}\left(\operatorname{Coker}\left(\beta^{\vee}\right), \mathbb{C}^{\times}\right)$, we have the exact sequence (1.1). Let $\Sigma(1)=$ $n$ be the set of one-dimensional cones in $\Sigma$ and $V \subset \mathbb{C}^{n}$ the closed subvariety defined by the ideal generated by

$$
J_{\Sigma}=\left\langle\prod_{\rho_{i} \nsubseteq \sigma \sigma} z_{i}: \sigma \in \Sigma\right\rangle .
$$

Let $Z:=\mathbb{C}^{n} \backslash V$. From [5], the complex codimension of $V$ in $\mathbb{C}^{n}$ is at least 2. The toric Deligne-Mumford stack $\mathcal{X}(\boldsymbol{\Sigma})=[Z / G]$ is the quotient stack where the action of $G$ is through the map $\alpha$ in (1.1).

Lemma 2.1. If $\operatorname{Codim}_{\mathbb{C}}\left(V, \mathbb{C}^{n}\right) \geq 2$, then $H^{1}(Z, \nu)=H^{2}(Z, \nu)=0$, where $\nu$ is a finite abelian group.

Proof. Consider the following exact sequence:

$$
\begin{aligned}
& 0 \longrightarrow H_{V}^{0}\left(\mathbb{C}^{n}, \nu\right) \longrightarrow H^{0}\left(\mathbb{C}^{n}, \nu\right) \longrightarrow H^{0}(Z, \nu) \longrightarrow \\
& \longrightarrow H_{V}^{1}\left(\mathbb{C}^{n}, \nu\right) \longrightarrow H^{1}\left(\mathbb{C}^{n}, \nu\right) \longrightarrow H^{1}(Z, \nu) \longrightarrow \\
& \longrightarrow H_{V}^{2}\left(\mathbb{C}^{n}, \nu\right) \longrightarrow \cdots
\end{aligned}
$$

Since $\operatorname{Codim}_{\mathbb{C}}\left(V, \mathbb{C}^{n}\right) \geq 2$, so the real codimension is at least 4 and $H_{V}^{i}\left(\mathbb{C}^{n}, \nu\right)=0$ for $i=1,2,3$, so from the exact sequence and $H^{i}\left(\mathbb{C}^{n}, \nu\right)=0$ for all $i>0$ we prove the lemma. 
2.1. The Proof of Theorem 1.1. Consider the following diagram:

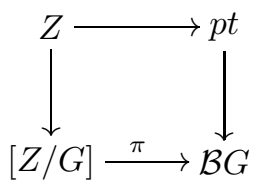

which is Cartesian. Consider the Leray spectral sequence for the fibration $\pi$ :

$$
H^{p}\left(\mathcal{B} G, R^{q} \pi_{*} \nu\right) \Longrightarrow H^{p+q}([Z / G], \nu) .
$$

We compute

$$
H^{2}([Z / G], \nu)=\bigoplus_{p+q=2} H^{p}\left(\mathcal{B} G, R^{q} \pi_{*} \nu\right) .
$$

First we have that $R^{q} \pi_{*} \nu=\left[H^{q}(Z, \nu) / G\right]$. There are three cases.

(1) When $p=2, q=0, R^{0} \pi_{*} \nu=\nu$ because $Z$ is connected, so

$$
H^{p}\left(\mathcal{B} G, R^{q} \pi_{*} \nu\right)=H^{2}(\mathcal{B} G, \nu) .
$$

(2) When $p=1, q=1, R^{1} \pi_{*} \nu=\left[H^{1}(Z, \nu) / G\right]$, so

$$
H^{p}\left(\mathcal{B} G, R^{q} \pi_{*} \nu\right)=H^{1}\left(\mathcal{B} G, H^{1}(Z, \nu)\right),
$$

and by Lemma 2.1, $H^{1}(Z, \nu)=0$, so we have $H^{p}\left(\mathcal{B} G, R^{q} \pi_{*} \nu\right)=0$.

(3) When $p=0, q=2, R^{2} \pi_{*} \nu=\left[H^{2}(Z, \nu) / G\right]$, so

$$
H^{p}\left(\mathcal{B} G, R^{q} \pi_{*} \nu\right)=H^{0}\left(\mathcal{B} G, H^{2}(Z, \nu)\right) ;
$$

also from Lemma 2.1, $H^{2}(Z, \nu)=0$, and so we have $H^{p}\left(\mathcal{B} G, R^{q} \pi_{*} \nu\right)=0$.

So we get that

$$
H^{2}([Z / G], \nu) \cong H^{2}(\mathcal{B} G, \nu)
$$

Since for the finite abelian group $\nu$, the $\nu$-gerbes are classified by the second cohomology group with coefficient in the group $\nu$, and Theorem 1.1 is proved.

2.2. The Proof of Corollary 1.2. Let $\mathcal{X}(\boldsymbol{\Sigma})=[Z / G]$. The $\nu$-gerbe $\mathcal{G}$ over $[Z / G]$ is induced from a $\nu$-gerbe $\mathcal{B} \widetilde{G}$ over $\mathcal{B} G$ in the following central extension:

$$
1 \longrightarrow \nu \longrightarrow \widetilde{G} \stackrel{\varphi}{\longrightarrow} G \longrightarrow 1,
$$

where $\widetilde{G}$ is an abelian group. So the pullback gerbe over $Z$ under the map $Z \longrightarrow$ $[Z / G]$ is trivial. So we have

$$
\mathcal{G}=\mathcal{B} \widetilde{G} \times{ }_{\mathcal{B} G}[Z / G]=[Z / \widetilde{G}] .
$$

The stack $[Z / \widetilde{G}]$ is this $\nu$-gerbe $\mathcal{G}$ over $[Z / G]$. Consider the commutative diagram:

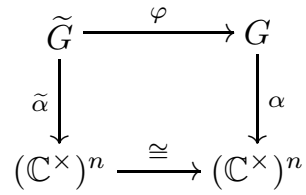

where $\alpha$ is the map in (1.1). So we have the following exact sequences:

$$
1 \longrightarrow \nu \longrightarrow \operatorname{ker}(\widetilde{\alpha}) \longrightarrow \mu \longrightarrow 1
$$

and

$$
1 \longrightarrow \operatorname{ker}(\widetilde{\alpha}) \longrightarrow \widetilde{G} \stackrel{\widetilde{\alpha}}{\longrightarrow}\left(\mathbb{C}^{\times}\right)^{n} \longrightarrow T \longrightarrow 1,
$$


where $T$ is the torus of the simplicial toric variety $X(\Sigma)$. Since the abelian groups $\widetilde{G}$, $G$ and $\left(\mathbb{C}^{\times}\right)^{n}$ are all locally compact topological groups, taking Pontryagin duality and the Gale dual, we have the following diagrams:

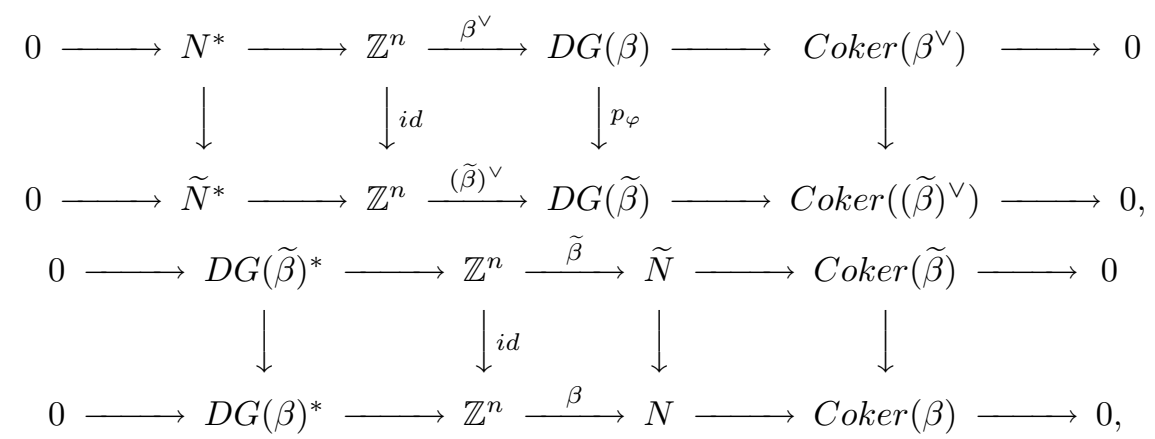

where $p_{\varphi}$ is induced by $\varphi$ in (2.1) under the Pontryagin duality. Suppose $\widetilde{\beta}$ : $\mathbb{Z}^{n} \longrightarrow \widetilde{N}$ is given by $\left\{\widetilde{b}_{1}, \cdots, \widetilde{b}_{n}\right\}$, then $\widetilde{\Sigma}:=(\widetilde{N}, \Sigma, \widetilde{\beta})$ is a new stacky fan. The toric Deligne-Mumford stack $\mathcal{X}(\widetilde{\boldsymbol{\Sigma}})=[Z / \widetilde{G}]$ is the $\nu$-gerbe $\mathcal{G}$ over $\mathcal{X}(\boldsymbol{\Sigma})$.

Remark 2.2. From Proposition 4.6 in [3, any Deligne-Mumford stack is a $\nu$-gerbe over an orbifold for a finite group $\nu$. Our results are the toric case of that general result.

In particular, given a stacky fan $\boldsymbol{\Sigma}=(N, \Sigma, \beta)$, let $\boldsymbol{\Sigma}_{\text {red }}=(\bar{N}, \Sigma, \bar{\beta})$ be the reduced stacky fan, where $\bar{N}$ is the abelian group $N$ modulo torsion, and $\bar{\beta}$ : $\mathbb{Z}^{n} \longrightarrow \bar{N}$ is given by $\left\{\bar{b}_{1}, \cdots, \bar{b}_{n}\right\}$, which are the images of $\left\{b_{1}, \cdots, b_{n}\right\}$ under the natural projection $N \longrightarrow \bar{N}$. Then the toric orbifold $\mathcal{X}\left(\boldsymbol{\Sigma}_{\text {red }}\right)=[Z / \bar{G}]$. From (1.1), let $\bar{G}=\operatorname{Im}(\alpha)$. Then we have the following exact sequences:

$$
\begin{gathered}
1 \longrightarrow \bar{G} \longrightarrow\left(\mathbb{C}^{\times}\right)^{n} \longrightarrow T \longrightarrow 1, \\
1 \longrightarrow \mu \longrightarrow G \longrightarrow \bar{G} \longrightarrow 1 .
\end{gathered}
$$

So $G$ is an abelian central extension of $\bar{G}$ by $\mu . \mathcal{X}(\boldsymbol{\Sigma})$ is a $\mu$-gerbe over the toric orbifold $\mathcal{X}\left(\boldsymbol{\Sigma}_{\text {red }}\right)$. Any $\mu$-gerbe over the toric orbifold coming from an abelian central extension is a toric Deligne-Mumford stack. This is a special case of the main results and is the toric case of rigidification construction in [1].

Remark 2.3. From the proof of Corollary 1.2 we see that if a $\nu$-gerbe over $\mathcal{X}(\boldsymbol{\Sigma})$ comes from a gerbe over $\mathcal{B} G$ and the central extension is abelian, then we can construct a new toric Deligne-Mumford stack.

\section{An example}

Example 3.1. Let $\Sigma$ be the complete fan of the projective line, $N=\mathbb{Z} \oplus \mathbb{Z} / 3 \mathbb{Z}$, and $\beta: \mathbb{Z}^{2} \longrightarrow \mathbb{Z} \oplus \mathbb{Z} / 3 \mathbb{Z}$ be given by the vectors $\left\{b_{1}=(1,0), b_{2}=(-1,1)\right\}$. Then $\boldsymbol{\Sigma}=(N, \Sigma, \beta)$ is a stacky fan. We compute that $(\beta)^{\vee}: \mathbb{Z}^{2} \longrightarrow D G(\beta)=\mathbb{Z}$ is given by the matrix $[3,3]$. So we get the following exact sequence:

$$
1 \longrightarrow \mu_{3} \longrightarrow \mathbb{C}^{\times} \stackrel{[3,3]^{t}}{\longrightarrow}\left(\mathbb{C}^{\times}\right)^{2} \longrightarrow \mathbb{C}^{\times} \longrightarrow 1 .
$$

The toric Deligne-Mumford stack is $\mathcal{X}(\boldsymbol{\Sigma})=\left[\mathbb{C}^{2}-\{0\} / \mathbb{C}^{\times}\right]$, where the action is given by $\lambda(x, y)=\left(\lambda^{3} x, \lambda^{3} y\right)$. So $\mathcal{X}(\boldsymbol{\Sigma})$ is the nontrivial $\mu_{3}$-gerbe over $\mathbb{P}^{1}$ coming 
from the canonical line bundle over $\mathbb{P}^{1}$. Let $\mathcal{G} \longrightarrow \mathcal{X}(\boldsymbol{\Sigma})$ be a $\mu_{2}$-gerbe such that it comes from the $\mu_{2}$-gerbe over $\mathcal{B} \mathbb{C}^{\times}$given by the central extension

$$
1 \longrightarrow \mu_{2} \longrightarrow \mathbb{C}^{\times} \stackrel{(\cdot)^{2}}{\longrightarrow} \mathbb{C}^{\times} \longrightarrow 1 .
$$

From the sequences (3.1) and (3.2), we have

$$
1 \longrightarrow \mu_{3} \otimes \mu_{2} \longrightarrow \mathbb{C}^{\times} \stackrel{[6,6]^{t}}{\longrightarrow}\left(\mathbb{C}^{\times}\right)^{2} \longrightarrow \mathbb{C}^{\times} \longrightarrow 1 .
$$

The Pontryagin dual of $\mathbb{C}^{\times} \stackrel{[6,6]^{t}}{\longrightarrow}\left(\mathbb{C}^{\times}\right)^{2}$ is $(\widetilde{\beta})^{\vee}: \mathbb{Z}^{2} \longrightarrow \mathbb{Z}$, which is given by the matrix $[6,6]$. Taking the Gale dual we have

$$
\widetilde{\beta}: \mathbb{Z}^{2} \longrightarrow \mathbb{Z} \oplus \mathbb{Z}_{6},
$$

which is given by the vectors $\left\{\widetilde{b}_{1}=(1,0), \widetilde{b}_{2}=(-1,1)\right\}$. Let $\widetilde{\Sigma}=(\widetilde{N}, \Sigma, \widetilde{\beta})$ be the new stacky fan. Then we have the toric Deligne-Mumford stack $\mathcal{X}(\widetilde{\boldsymbol{\Sigma}})=$ $\left[\mathbb{C}^{2}-\{0\} / \mathbb{C}^{\times}\right]$, where the action is given by $\lambda(x, y)=\left(\lambda^{6} x, \lambda^{6} y\right)$. So $\mathcal{X}(\widetilde{\boldsymbol{\Sigma}})$ is the canonical $\mu_{6}$-gerbe over $\mathbb{P}^{1}$.

If the $\mu_{2}$-gerbe over $\mathcal{B} \mathbb{C}^{\times}$is given by the central extension

$$
1 \longrightarrow \mu_{2} \longrightarrow \mathbb{C}^{\times} \times \mu_{2} \stackrel{\alpha}{\longrightarrow} \mathbb{C}^{\times} \longrightarrow 1
$$

where $\alpha$ is given by the matrix $[1,0]$, then from (3.1) and (3.3), we have

$$
1 \longrightarrow \mu_{3} \otimes \mu_{2} \longrightarrow \mathbb{C}^{\times} \times \mu_{2} \stackrel{\varphi}{\longrightarrow}\left(\mathbb{C}^{\times}\right)^{2} \longrightarrow \mathbb{C}^{\times} \longrightarrow 1,
$$

where $\varphi$ is given by the matrix $\left[\begin{array}{ll}3 & 0 \\ 3 & 0\end{array}\right]$. The Pontryagin dual of $\varphi$ is: $\left(\widetilde{\beta}^{\prime}\right)^{\vee}$ : $\mathbb{Z}^{2} \longrightarrow \mathbb{Z} \oplus \mathbb{Z}_{2}$, which is given by the transpose of the above matrix. Taking the Gale dual we get

$$
\widetilde{\beta}^{\prime}: \mathbb{Z}^{2} \longrightarrow \mathbb{Z} \oplus \mathbb{Z}_{3} \oplus \mathbb{Z}_{2}
$$

which is given by the vectors $\left\{\widetilde{b}_{1}=(1,0,0), \widetilde{b}_{2}=(-1,1,0)\right\}$. So $\widetilde{\boldsymbol{\Sigma}}^{\prime}=\left(\widetilde{N}^{\prime}, \Sigma, \widetilde{\beta}^{\prime}\right)$ is a stacky fan. The toric Deligne-Mumford stack is $\mathcal{X}\left(\widetilde{\boldsymbol{\Sigma}}^{\prime}\right)=\left[\mathbb{C}^{2}-\{0\} / \mathbb{C}^{\times} \times \mu_{2}\right]$, where the action is $\left(\lambda_{1}, \lambda_{2}\right) \cdot(x, y)=\left(\lambda_{1}^{3} x, \lambda_{1}^{3} y\right)$. So $\mathcal{G}^{\prime}=\mathcal{X}\left(\widetilde{\boldsymbol{\Sigma}}^{\prime}\right)$ is the trivial $\mu_{2}$-gerbe over $\mathcal{X}(\boldsymbol{\Sigma})$ and $\mathcal{X}(\widetilde{\boldsymbol{\Sigma}}) \nsucceq \mathcal{X}\left(\widetilde{\boldsymbol{\Sigma}}^{\prime}\right)$.

\section{ACKNowledgments}

I would like to thank the referee for nice comments about the proof of the main results. I thank my advisor, Kai Behrend, for encouragement and Hsian-Hua Tseng for valuable discussions.

\section{REFERENCES}

1. D. Abramovich, A. Corti and A. Vistoli, Twisted bundles and admissible covers, Commun. Algebra 31 (2003), no. 8, 3547-3618. MR2007376 (2005b:14049)

2. K. Behrend, D. Edidin, B. Fantechi, W. Fulton, L. Göttsche, and A. Kresch, Introduction to stacks, in preparation.

3. K. Behrend and B. Noohi, Uniformization of Deligne-Mumford curves, J. Reine Angew. Math. 599 (2006), 111-153. MR2279100 (2007k:14017)

4. L. Borisov, L. Chen and G. Smith, The orbifold Chow ring of toric Deligne-Mumford stacks, J. Amer. Math. Soc. 18 (2005), no. 1, 193-215. MR2114820(2006a:14091) 
5. D. Cox, The homogeneous coordinate ring of a toric variety, J. of Algebraic Geometry, 4 (1995), 17-50. MR1299003 (95i:14046)

6. W. Fulton, Introduction to toric varieties, Annals of Mathematics Studies 131, Princeton University Press, Princeton, NJ, 1993. MR1234037 (94g:14028)

Department of Mathematics, University of Utah, 155 South 1400 East JWB233, Salt LAKe City, Utah 84112

E-mail address: jiangyf@math.utah.edu 